\title{
SAPONIFICAÇÃO ASSISTIDA POR MICRO-ONDAS NA EXTRAÇÃO DE DITERPENOS EM CAFÉ ARÁBICA TORRADO
}

\author{
Mirelli Bianchin*, Fabio Yamashita e Marta de Toledo Benassi \\ Departamento de Ciência e Tecnologia de Alimentos, Universidade Estadual de Londrina, 86057-970 Londrina - PR, Brasil
}

Recebido em 07/05/2017; aceito em 20/07/2017; publicado na web em 12/09/2017

\begin{abstract}
MICROWAVE ASSISTED SAPONIFICATION FOR DITERPENES EXTRACTION IN ROASTED ARABICA COFFEE. The extraction of kahweol and cafestol involves saponification reaction. This step is important for the efficiency of the extraction as the diterpenes are susceptible to structural changes during hydrolysis. The microwave-assisted saponification has been successfully used in different matrices, but there is no previous report of the use of this technique in the saponification of diterpenes. Therefore, the effect of microwave-assisted saponification on the extraction of kahweol and cafestol in roasted coffee was evaluated. A $3^{2}$ factorial experimental design was used in order to evaluate the effect of temperature $\left(70,80\right.$, and $\left.90^{\circ} \mathrm{C}\right)$ and reaction time $(4,8$, and $12 \mathrm{~min})$. The quantification of diterpenes was performed by high-performance liquid chromatography and the results were compared to with those obtained by a method, which uses thermostatic bath for saponification. Temperature and time had a significant effect $(\mathrm{p}<0.05)$ on diterpenes levels. For simultaneous saponification of kahweol and cafestol, the optimal reaction conditions were $80{ }^{\circ} \mathrm{C} / 12 \mathrm{~min}$. The use of microwave-assisted procedure lead to lower yields of kahweol (24\%) and cafestol (35\%) compared to reference method. However a significant reduction of reaction time $(80 \%)$ was achieved, and the procedure also has the advantage of controlled process conditions and the possibility of extended scale.
\end{abstract}

Keywords: kahweol; cafestol; microwave assisted saponification; Coffea arabica.

\section{INTRODUÇÃo}

O café é uma das bebidas mais populares do mundo e a produção e comercialização do grão representa uma atividade econômica importante para muitos países, incluindo o Brasil, que é o maior produtor mundial de café e o segundo maior consumidor. ${ }^{1}$ As espécies de café mais importantes comercialmente são o Coffea canephora Pierre ex A. Froehner e o Coffea arabica L. No Brasil, o café arábica representa $80 \%$ da produção total de café. ${ }^{2}$

Os lipídios constituem uma das classes de compostos mais importantes no café e estão relacionados com a qualidade e as propriedades funcionais da bebida. ${ }^{3}$ Benefícios à saúde decorrentes do consumo moderado de café, como a redução da incidência de doenças crônico-degenerativas, têm sido reportados, assim como a correlação do consumo da bebida com a redução da taxa de mortalidade. ${ }^{4} \mathrm{~A}$ fração lipídica do café é composta principalmente por triacilgliceróis, esteróis e quantidades significativas de diterpenos com esqueleto do tipo caurano. O caveol (PubChem CID: 114778) e cafestol (PubChem CID: 108052) (Figura 1), principais representantes dos diterpenos no café, correspondem a $20 \%$ dos lipídios no grão e podem ser encontrados na forma livre ou esterificados com diferentes ácidos graxos, sendo a segunda forma a majoritária. ${ }^{5-7} \mathrm{O}$ interesse por estes compostos é reportado em diferentes estudos que investigam desde a contribuição à saúde pelo consumo de bebidas de café até a aplicação como parâmetro de qualidade, para identificação de espécies de café em blends comerciais. ${ }^{8}$ Bioativos de importância pela reconhecida capacidade antioxidante e as propriedades anticarcinogênicas e anti-inflamatórias, tem sido ainda citada a aplicação de diterpenos em produtos cosméticos. ${ }^{8}$ Considerando a dificuldade de síntese dos diterpenos e a restrita disponibilidade de compra e o alto preço dos padrões comerciais, metodologias eficientes para extração desses compostos, que permitam posterior purificação, são também de interesse. ${ }^{9}$

\footnotetext{
*e-mail: bianchin.mirelli@gmail.com
}

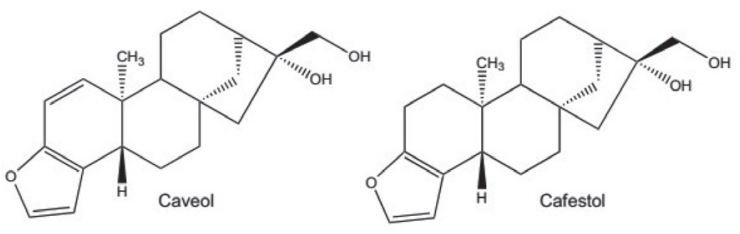

Figura 1. Estruturas químicas do caveol e cafestol

A maior proporção dos diterpenos do café encontra-se na forma esterificada, assim, para a extração de caveol e cafestol é necessário que ocorra uma etapa de hidrólise, como a saponificação ou a metanólise para a obtenção dos diterpenos livres. ${ }^{10,11}$ Nos métodos que utilizam a saponificação, o tempo da reação varia de 1 a 4 h, o que torna o processo exaustivo e limitado. Portanto, é importante desenvolver novas metodologias para extração de caveol e cafestol, para a posterior aplicação destes compostos em potencial.

A literatura reporta duas abordagens para separação de diterpenos em café: a extração prévia dos lipídios seguida de saponificação, ${ }^{5,12-14}$ ou metanólise, ${ }^{10,15}$ com posterior separação dos compostos ou a saponificação direta seguida de separação com solvente orgânico. ${ }^{16-22}$ Dias et al. ${ }^{18}$ estudando extração de diterpenos de café, reportaram uma maior eficiência da saponificação à quente (em banho à $80^{\circ} \mathrm{C}$, $1 \mathrm{~h}$ ) comparativamente a saponificação à frio e a extração (Soxhlet ou Bligh \& Dyer) seguida de saponificação à quente.

A utilização de aquecimento em banho térmico apresenta limitações quando há o interesse em adaptar procedimentos de um método analítico ${ }^{18}$ para uma extração em maior escala. Uma ampliação da proporção amostra/solvente aumentaria ainda mais o tempo da reação de saponificação e restringiria a utilização do aquecimento convencional, devido às zonas irregulares de aquecimento que são formadas durante a transferência de calor por condução e convecção. ${ }^{23}$ Além disto, o caveol e o cafestol apresentam sensibilidade a ácidos, bases e oxidantes, a qual está relacionada à presença do anel furano. ${ }^{24,25}$ Portanto, o controle da etapa de saponificação é muito importante, uma vez que durante a hidrólise os compostos ficam susceptíveis à 
mudanças na estrutura, ${ }^{6,15}$ assim, condições de estresse como variações de temperatura devem ser evitadas. ${ }^{10}$

Como alternativa aos métodos convencionais de aquecimento, a utilização do aquecimento por irradiação de micro-ondas é considerada uma técnica promissora por permitir a extração de diferentes compostos e melhorar processos químicos. ${ }^{26-28}$ Entre as vantagens do uso de micro-ondas em reações químicas em relação ao aquecimento convencional destacam-se a redução no tempo e aumento da taxa de reação, o aumento do rendimento e da seletividade e o menor consumo de energia, permitindo realizar transformações mais seguras, com menor produção de resíduos e em um maior número de amostras. ${ }^{29,30}$ O emprego de uma reação de saponificação mais eficiente, e em maior escala, poderia viabilizar a extração de diterpenos da matriz de café torrado, permitindo seu posterior uso em produtos de alimentos ou farmacêuticos, ou, após purificação, como padrões analíticos.

Não há informações na literatura sobre o uso da energia de micro-ondas na reação de saponificação para extração de diterpenos. Assim, este trabalho teve como objetivo estudar os efeitos dos parâmetros de tempo e temperatura na saponificação assistida por micro-ondas para extração de caveol e cafestol em café torrado. A eficiência do processo de saponificação em micro-ondas foi verificada através do teor de caveol e cafestol extraído. Após a definição da melhor condição de processo, os resultados foram comparados aos obtidos utilizando como referência o método de saponificação em banho térmico. ${ }^{18}$

\section{EXPERIMENTAL}

A proposta da metodologia de saponificação em micro-ondas foi baseada e adaptada do método estabelecido por Dias et al. ${ }^{18}$ que consiste na saponificação direta à quente da fração lipídica do café em banho termostático a $80^{\circ} \mathrm{C}$ durante $1 \mathrm{~h}$, seguida da separação da fração insaponificável por separação líquido/líquido e quantificação dos diterpenos por cromatografia líquida de alta eficiência (CLAE).

\section{Reagentes e padrões}

Utilizou-se como reagentes para a etapa de saponificação: hidróxido de potássio $(\mathrm{KOH})$ 85\% (Synth, Diadema, São Paulo, Brasil) e etanol P.A. (Impex, Varginha, Minas Gerais, Brasil). Para a separação dos compostos e análise cromatográfica foram empregados terc-butil metil éter 99,5\% (Sigma Aldrich, Steinheim, Alemanha), acetonitrila grau HPLC (Panreac, Barcelona, Espanha), água purificada (Sistema de purificação Milli-Q®, Millipore, Billerica, EUA) e padrões analíticos de caveol e cafestol (pureza $>98 \%$, Axxora, San Diego, EUA) certificados pela Alexis Biochemicals (Lausanne, Suíça).

\section{Material}

As amostras de café arábica torrado comercial em grão (Spresso Melitta, Avaré, São Paulo) foram caracterizadas quanto à granulometria e umidade. O café foi moído em moedor (moinho Krups GVX 2, China) até a granulometria de 16 mesh. A umidade (4,95 g 100 $\mathrm{g}^{-1}$ ) foi determinada em equipamento de infravermelho (OHAUS, Parsippany, EUA) à $105{ }^{\circ} \mathrm{C}$ durante $7 \mathrm{~min},{ }^{18} \mathrm{e}$ o resultado obtido foi utilizado para os cálculos do teor de caveol e cafestol em base seca.

\section{Métodos}

Reação de saponificação assistida por micro-ondas

A reação de saponificação foi realizada em equipamento de micro-ondas (Ethos One, Milestone, Itália), sob agitação fixa a 700 W. Em frasco de Teflon, adicionou- se $10 \mathrm{~mL}$ de hidróxido de potássio (2,5 mol L $\mathrm{L}^{-1}$ ) em etanol (96\%) a 1,0 g de café torrado e moído. Optou-se em manter a proporção original de 1:10 (m/v), utilizada por Dias et al. ${ }^{18}$ mas ampliando-se a escala em cinco vezes. A reação de saponificação foi conduzida de acordo com as variações das condições de tempo e temperatura estipuladas pelo planejamento fatorial.

O perfil de aquecimento consistiu em uma rampa de aquecimento linear. A temperatura desejada $\left(70,80\right.$ e $\left.90^{\circ} \mathrm{C}\right)$ foi alcançada em 2 min e manteve-se durante o tempo de reação (4, 8 e 12 min), seguido de um tempo de exaustão de $2 \mathrm{~min}$, no qual o interior do equipamento atinge $65 \pm 3^{\circ} \mathrm{C}$. O equipamento de micro-ondas foi operado em modo de controle de temperatura, monitorado por sensor infravermelho.

\section{Reação de saponificação em aquecimento convencional}

Para saponificação em banho utilizou-se o método de referência, ${ }^{18}$ no qual $200 \mathrm{mg}$ de amostra e $2 \mathrm{~mL}$ de hidróxido de potássio (2,5 mol $\left.\mathrm{L}^{-1}\right)$ em etanol (96\%) foram submetidos ao aquecimento $\left(80^{\circ} \mathrm{C}\right) \mathrm{em}$ banho termostatizado (MA 127/BO, Marconi, Piracicaba, São Paulo, Brasil) durante $1 \mathrm{~h}$.

\section{Extração do caveol e cafestol e preparo das amostras}

A extração e o preparo das amostras foram realizados conforme descrito por Dias et al. ${ }^{18}$ Adaptaram-se os volumes de solvente devido a ampliação da escala em 5 vezes. Após a saponificação em micro-ondas, as amostras foram transferidas para tubos de centrífuga e adicionou-se $10 \mathrm{~mL}$ água destilada e $10 \mathrm{~mL}$ de terc-butil metil éter para a extração da fração insaponificável. Nas amostras saponificadas em banho, seguiu-se o mesmo procedimento, somente alterando o volume de $10 \mathrm{~mL}$ dos solventes para $2 \mathrm{~mL}$. Em seguida, as amostras foram agitadas e centrifugadas (2 min a $3000 \mathrm{rpm}$ ) (Sovall SS-3, Ivan Sorvall, Nova Iorque, EUA). Para a coleta da fase orgânica, o processo de adição de solvente orgânico e centrifugação foi repetido três vezes. Após limpeza da fração insaponificável com água destilada (10-2 mL) o extrato orgânico foi coletado e o solvente evaporado em banho termostatizado a $70^{\circ} \mathrm{C}$ (MA 127/BO, Marconi, Piracicaba, São Paulo, Brasil). O extrato foi ressuspendido em fase móvel, filtrado em membrana de PVDF Millex ${ }^{\circledR}$ 0,22 $\mu \mathrm{m}$ (Millipore, São Paulo, Brasil) e acondicionado em tubos vials.

\section{Análise cromatográfica}

Para quantificação dos diterpenos, utilizou-se Cromatógrafo Líquido de Alta Eficiência (Shimadzu, Kyoto, Japão), com bomba com gerenciador de solvente quaternário e desgaseificador (DGU 20 $\mathrm{A}_{\mathrm{S}}$ ), auto amostrador (SIL20HT), válvula injetora (LC20AT) com alça de amostragem, forno para coluna (CTO 20A), detector UV/Vis (SPDM20A), interface e sistema de dados controlado pelo software LC solution. A análise cromatográfica foi adaptada de Dias et al. ${ }^{18} \mathrm{e}$ Mori et al. ${ }^{22}$ Utilizou-se uma coluna Kinetex $2.6 \mu \mathrm{m} \mathrm{C18}(150 \mathrm{~mm}$ x 4,6 mm) (Phenomenex, Torrance, EUA), eluição isocrática com fase móvel na proporção de 3:2 de água e acetonitrila, vazão de $0,4 \mathrm{~mL}$ $\min ^{-1}$, volume de injeção de $0,400 \mu \mathrm{L}$ e detecção a $230 \mathrm{~nm}$ (cafestol) e $290 \mathrm{~nm}$ (caveol). A temperatura da coluna foi mantida a $55{ }^{\circ} \mathrm{C}$ e o tempo de eluição foi 30 min. A injeção das amostras foi feita em triplicata e a identificação dos compostos foi realizada com base nos tempos de retenção dos padrões e espectro no UV/Vis (Material Suplementar, Figura 1S). A quantificação foi feita por padronização externa utilizando padrões comerciais, construindo-se curvas analíticas de 7 pontos, em triplicata, na faixa de 30 a $200 \mu \mathrm{g} \mathrm{mL}^{-1}\left(\mathrm{R}^{2} \geq\right.$ $0,99, \mathrm{p}<0,001)$. O teor dos diterpenos foi expresso em base seca (bs).

\section{Delineamento experimental e análise dos resultados}

Para a etapa de saponificação em micro-ondas, utilizou-se como delineamento experimental um planejamento fatorial completo $3^{2}$, totalizando 9 ensaios (Tabela 1), que foram conduzidos de forma 
aleatória. As extrações foram realizadas em triplicata. Como variáveis independentes foram estudadas a temperatura $\left(\mathrm{X}_{1}\right)$ e tempo $\left(\mathrm{X}_{2}\right)$, e como variáveis dependentes (y), o teor de caveol e cafestol. Os níveis escolhidos para tempo de reação (4, 8 e $12 \mathrm{~min}$ ) foram baseados na literatura, ${ }^{15,31,32}$ e para temperatura $\left(70,80\right.$ e $\left.90{ }^{\circ} \mathrm{C}\right)$, definiu-se como o ponto central $\left(80^{\circ} \mathrm{C}\right)$ a temperatura proposta na metodologia de referência. ${ }^{18}$

Os resultados obtidos pelo planejamento foram analisados utilizando o software Statistica ${ }^{\mathrm{TM}}$ Version 7 (Statsoft, Tulsa, EUA), ${ }^{33}$ gerando um modelo polinomial de segunda ordem (Equação 1).

$y=B_{0}+B_{1} X_{1}+B_{2} X_{2}+B_{11} X_{1}^{2}+B_{22} X_{2}^{2}+B_{12} X_{1} X_{2}+B_{1} B_{22} X_{1} X_{2}^{2}+$ $B_{11} B_{2} X_{1}^{2} X_{2}+B_{11} B_{22} X_{1}^{2} X_{2}^{2}$

em que $B_{1}, B_{2}$, correspondem aos coeficientes de regressão linear, $B_{11}, B_{22}$, os coeficientes de regressão quadrática, $B_{12}$, a interação entre os coeficientes lineares do modelo, $B_{1} B_{22} e B_{11} B_{2}$ as interações entre termos lineares e quadráticos e $B_{11} B_{22}$ as interações entre termos quadráticos.

Os efeitos lineares e quadráticos dos fatores e suas interações foram estudados para cada composto, utilizando análise de variância (ANOVA) ao nível de $95 \%$ de confiança. A função desejabilidade foi empregada para maximizar conjuntamente os teores dos diterpenos utilizando o software Statistica ${ }^{\mathrm{TM}}$ Version 7 (Statsoft, Tulsa, EUA). ${ }^{33}$

Após definir a melhor condição de saponificação em micro-ondas, os teores de caveol e cafestol foram comparados aos obtidos por saponificação em banho térmico utilizando o método de referência. ${ }^{18}$ Para complementar a informação sobre a influência do tempo na saponificação, realizou-se um estudo do perfil do teor de diterpenos obtido na melhor temperatura de saponificação em função do tempo de reação. Para a etapa utilizando micro-ondas, empregaram-se os tempos de 4, 8, 12, 16, 20 e 24 min, e para o banho convencional, 5, $10,20,40,60,80$ e $100 \mathrm{~min}$.

\section{RESULTADOS E DISCUSSÃO}

Conforme o delineamento experimental, os teores de caveol e cafestol obtidos por CLAE após saponificação em micro-ondas variaram de 314 a 474 mg $100 \mathrm{~g}^{-1}$ e de 250 a $373 \mathrm{mg} 100 \mathrm{~g}^{-1}$, respectivamente (Tabela 1). Para a saponificação conduzida em banho, utilizada como

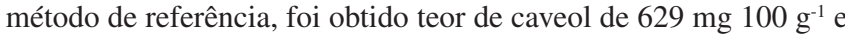

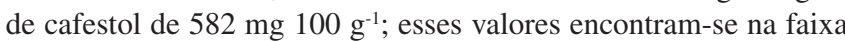
descrita na literatura para café arábica brasileiro. ${ }^{11,34-36}$

Tabela 1. Teores de caveol e cafestol em café arábica torrado obtidos por CLAE após saponificação em micro-ondas conforme delineamento fatorial completo $3^{2}$

\begin{tabular}{|c|c|c|c|c|}
\hline \multirow{2}{*}{ Ensaio } & \multicolumn{2}{|c|}{ Variáveis } & \multirow{2}{*}{$\begin{array}{c}\text { Caveol }^{\mathrm{a}} \\
\left(\mathrm{mg} 100 \mathrm{~g}^{-1}\right)\end{array}$} & \multirow{2}{*}{$\begin{array}{c}\text { Cafestol }^{\mathrm{a}} \\
\left(\mathrm{mg} 100 \mathrm{~g}^{-1}\right)\end{array}$} \\
\hline & Temperatura $\left({ }^{\circ} \mathrm{C}\right)$ & Tempo (min) & & \\
\hline 1 & 70 & 4 & $314 \pm 9$ & $250 \pm 7$ \\
\hline 2 & 70 & 8 & $362 \pm 8$ & $287 \pm 5$ \\
\hline 3 & 70 & 12 & $364 \pm 14$ & $304 \pm 4$ \\
\hline 4 & 80 & 4 & $393 \pm 12$ & $311 \pm 6$ \\
\hline 5 & 80 & 8 & $388 \pm 5$ & $310 \pm 5$ \\
\hline 6 & 80 & 12 & $474 \pm 16$ & $373 \pm 14$ \\
\hline 7 & 90 & 4 & $352 \pm 6$ & $277 \pm 5$ \\
\hline 8 & 90 & 8 & $407 \pm 9$ & $301 \pm 11$ \\
\hline 9 & 90 & 12 & $333 \pm 10$ & $261 \pm 8$ \\
\hline
\end{tabular}

${ }^{a}$ Média \pm desvio padrão (3 repetições de processo).
Através da tabela de efeitos (Tabela 2), observa-se que a temperatura e o tempo são parâmetros importantes, mas que exercem influência diferente sobre cada composto. As tabelas de ANOVA correspondentes estão disponíveis no Material Suplementar (Tabelas 1S e 2S).

Tabela 2. Efeitos das variáveis temperatura $\left(X_{1}\right)$ e tempo $\left(X_{2}\right)$ na reação de saponificação por micro-ondas para obtenção de caveol e cafestol em café arábica torrado

\begin{tabular}{lcccc}
\hline \multirow{2}{*}{ Fatores } & \multicolumn{2}{c}{ Caveol } & \multicolumn{2}{c}{ Cafestol } \\
\cline { 2 - 5 } & Efeito & p- valor & Efeito & p- valor \\
\hline Média & 385,9 & $<0,001$ & 300,8 & $<0,001$ \\
Temperatura (L) & 44,73 & $<0,001$ & n.s & $>0,05$ \\
Temperatura (Q) & n.s & $>0,05$ & $-25,72$ & 0,079 \\
Tempo (L) & 81,16 & $<0,001$ & 55,81 & $<0,001$ \\
Tempo (Q) & 95,27 & $<0,001$ & 89,90 & $<0,001$ \\
Temperatura (L)*Tempo (L) & $-34,26$ & 0,005 & $-33,33$ & $<0,001$ \\
Temperatura (L)*Tempo (Q) & $-41,62$ & $<0,001$ & n.s & $>0,05$ \\
Temperatura (Q)*Tempo (L) & $-65,60$ & $<0,001$ & $-38,17$ & 0,003 \\
Temperatura (Q)*Tempo (Q) & $-185,58$ & $<0,001$ & $-140,62$ & $<0,001$ \\
\hline
\end{tabular}

n.s - não significativo ao nível de $95 \%$ de confiança (p-valor $>0,05$ ), (L) - termo linear, $(\mathrm{Q})$ - termo quadrático.

Com base nos fatores significativos $(\mathrm{p}<0,05)$ gerou-se o modelo matemático que descreve o efeito da temperatura $\left(\mathrm{X}_{1}\right)$ e do tempo $\left(\mathrm{X}_{2}\right)$ sobre a extração de caveol e cafestol (Equações 2 e 3 ) na reação de saponificação em micro-ondas. Obtiveram-se coeficientes de determinação $\left(\mathrm{R}^{2}\right)$ de 0,94 para a extração do caveol e de 0,91 para o cafestol, indicando boa qualidade no ajuste dos dois modelos.

Caveol $=385,9+22,36 X_{1}+40,57 X_{2}+47,63 X_{2}^{2}-17,13 X_{1} X_{2}-$ $20,80 X_{1} X_{2}^{2}-32,75 X_{1}^{2} X_{2}-92,80 X_{1}^{2} X_{2}^{2}$

Cafestol $=300,8+27,9 X_{2}+44,95 X_{2}^{2}-16,7 X_{1} X_{2}-19,08 X_{1}^{2} X_{2}-$ $70,31 X_{1}^{2} X_{2}^{2}$

Para o caveol, constatou-se que a temperatura (termo linear) e o tempo (termo linear e quadrático) exerceram efeito positivo; para o cafestol, o tempo (termo linear) também apresentou efeito positivo $(\mathrm{p}<0,05)$, porém a temperatura não teve efeito significativo ( $\mathrm{p}>$ 0,05) (Tabela 2, Eq. 2 e 3). As demais interações, para ambos os compostos, apresentaram efeito negativo, o que sugere a presença de uma faixa satisfatória de estudo, na qual atinge-se o máximo do teor de caveol e cafestol, seguido de redução, devido à formação de pontos de inflexão (Tabela 2, Equação 2 e 3, Figura 2). Esse comportamento demonstra que o tempo e a temperatura são parâmetros importantes para a reação de saponificação e que as interações entre eles são limitantes no processo.

Observou-se que para o caveol e cafestol a região ótima de saponificação (Figuras 2A e 2B) ocorreu próximo ao tempo em nível superior (12 $\mathrm{min})$ e em temperatura próxima ao ponto central $\left(80^{\circ} \mathrm{C}\right)$. Para cafestol a temperatura de $80^{\circ} \mathrm{C}$ foi a melhor, independentemente do tempo de reação. Para o caveol, a utilização de tempos próximos ao ponto central necessita do uso de temperaturas mais elevadas $\left(>80^{\circ} \mathrm{C}\right)$.

Para os dois compostos, observou-se efeito positivo com aumento da temperatura até $80{ }^{\circ} \mathrm{C}$. Em temperaturas mais elevadas ocorre a diminuição da tensão superficial e da viscosidade do solvente, o que facilita a penetração do solvente na matriz e favorece o rompimento celular, permitindo que os compostos fiquem mais disponíveis para extração. ${ }^{37} \mathrm{Com}$ o uso de temperaturas menores que $80{ }^{\circ} \mathrm{C}$, mesmo 

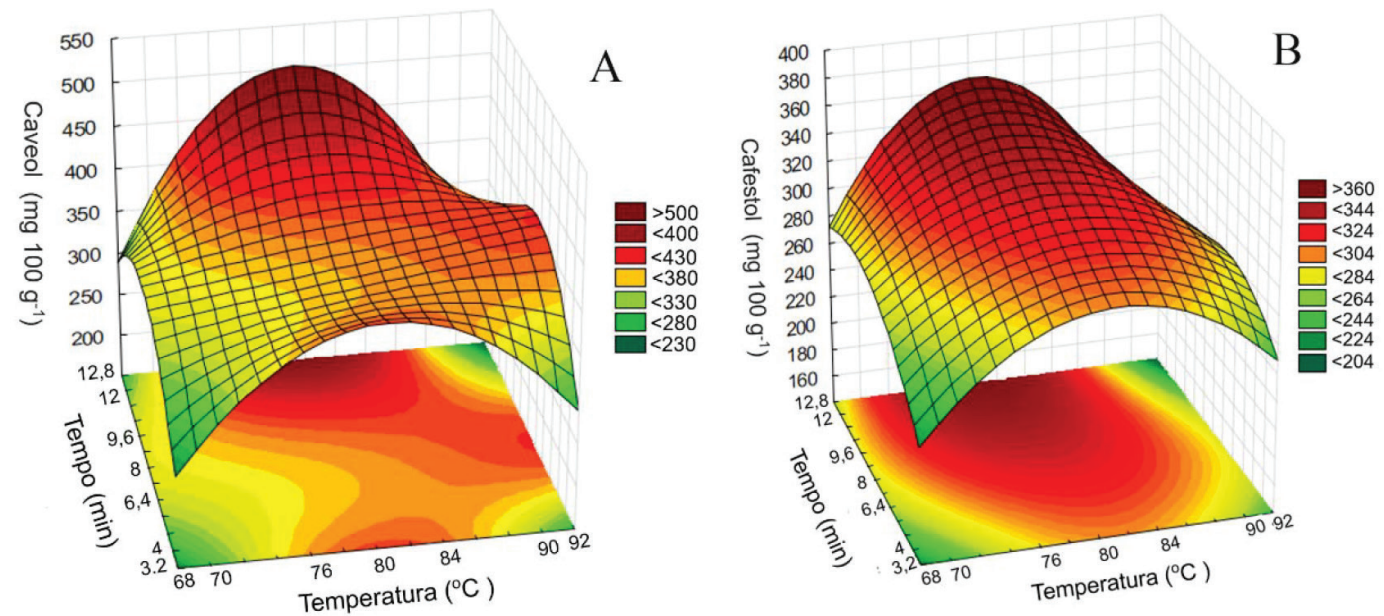

Figura 2. Superfícies de resposta para teores de caveol (A) e cafestol (B) obtidos por saponificação em micro-ondas sob diferentes condições de temperatura e tempo

por tempos prolongados, não foi observada a mesma eficiência, indicando que essas condições provavelmente não são suficientes para saponificar os ésteres de ácidos graxos diterpênicos.

No entanto, a utilização de temperaturas superiores a $80^{\circ} \mathrm{C}$ também não apresentou bons resultados, com redução do rendimento dos dois diterpenos. Embora os diterpenos tenham relativa estabilidade até mesmo a processos agressivos como o de torra, ${ }^{38}$ durante a reação de saponificação estes compostos ficam suscetíveis a mudanças na estrutura, podendo ser degradados. ${ }^{15}$ Além disto, Chartier et al. ${ }^{39}$ descrevem que a hidrólise é uma etapa crítica devido à sensibilidade do radical furano, e que essa sensibilidade está relacionada aos processos de aquecimento normalmente usados para obter os diterpenos.

O método de saponificação a quente desenvolvido por Dias et al. ${ }^{18}$ foi baseado no trabalho de Urgert et al. ${ }^{16}$, que propuseram a saponificação de café torrado a $80^{\circ} \mathrm{C}$ durante uma hora, porém a relação entre a reação de saponificação e a temperatura específica de $80^{\circ} \mathrm{C}$ não é reportada. Chartier et $a l .{ }^{39}$ verificaram que a melhor condição de transesterificação para obtenção de caveol e cafestol em óleo de café arábica verde ocorreu na temperatura de $70^{\circ} \mathrm{C}$. Oigman et al. ${ }^{10}$ descrevem que para obtenção dos diterpenos em óleo de café arábica verde por reação de metanólise em micro-ondas, a melhor temperatura foi de $100{ }^{\circ} \mathrm{C}$. Desta forma verifica-se que, para a separação dos diterpenos dos ésteres de ácidos graxos, a temperatura ótima depende do tipo de reação e do tipo de processo utilizado.

Observou-se que o efeito da temperatura foi maior para a extração do caveol que para a do cafestol, provavelmente devido a diferenças na estrutura dos compostos. Oigman et al. ${ }^{10} \mathrm{e}$ Chartier et al. ${ }^{39}$ reportam que em reações de hidrólise, dependo das condições, ambos os diterpenos são afetados, porém o impacto sob caveol é maior do que sobre o cafestol.

$\mathrm{O}$ cromatograma correspondente à saponificação a $80{ }^{\circ} \mathrm{C} \mathrm{em}$ micro-ondas (Figura 3A) mostra um perfil similar ao obtido por saponificação em banho (Figura 3B) na mesma temperatura. Apesar do aquecimento em micro-ondas ter ocorrido mais rapidamente, não foram observados picos de interferentes/produtos de degradação. Oigman et al. ${ }^{10}$ e Tsukui et al. ${ }^{15}$ na extração de diterpenos por metanólise e Liu et al. ${ }^{40}$ na extração de trigonelina, ácido nicotínico e cafeína em café arábica também não observaram formação de interferentes nas amostras submetidas a irradiação por micro-ondas.

Considerando o interesse em maximizar a extração conjunta dos diterpenos, utilizou-se a função de desejabilidade global, uma técnica de otimização simultânea, que indicou que a melhor condição de saponificação ocorreu em $80^{\circ} \mathrm{C}$ durante 12 min (Figura 4). Nessa condição, foram obtidos teores de caveol e cafestol de $474 \pm 16$ e

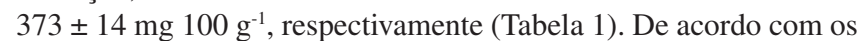
perfis de desejabilidade de tempo e temperatura (Figura 4), é possível observar que a temperatura foi otimizada, uma vez que temperaturas acima ou abaixo de $80{ }^{\circ} \mathrm{C}$ diminuem a quantidade extraída tanto de caveol quanto de cafestol. Entretanto, o aumento do tempo de processo poderia aumentar o conteúdo de diterpenos, pois $12 \mathrm{~min}$ foi o tempo máximo utilizado no planejamento fatorial, apesar da tendência ser de estabilização próximo a 12 min.

Para isto estudou-se o perfil de saponificação em micro-ondas para cada composto (Figura 5A), fixando-se a temperatura da reação em $80{ }^{\circ} \mathrm{C}$ e variando-se o tempo de 4 até 24 min. Para fins comparativos, estudou-se o perfil de saponificação em banho térmico, na
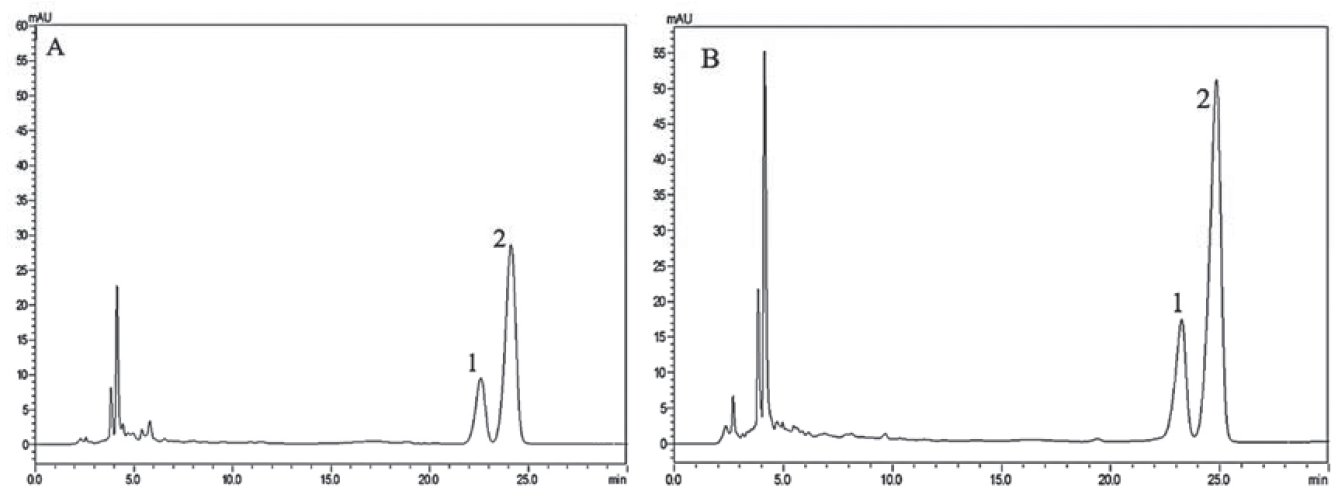

Figura 3. Cromatogramas dos diterpenos, caveol e cafestol, em café arábica torrado obtidos após a saponificação em micro-ondas $\left(80{ }^{\circ} \mathrm{C} / 12 \mathrm{~min}\right)(\mathrm{A})$ e em banho térmico $\left(80^{\circ} \mathrm{C} / 60 \mathrm{~min}\right)(B)$. Detecção: $230 \mathrm{~nm}$. Picos: caveol (1) e cafestol (2) 

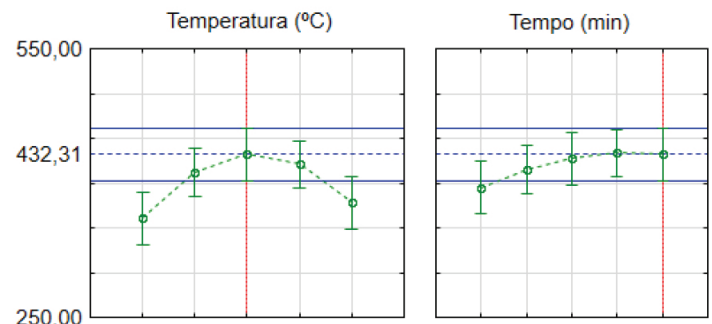

Desejabilidade
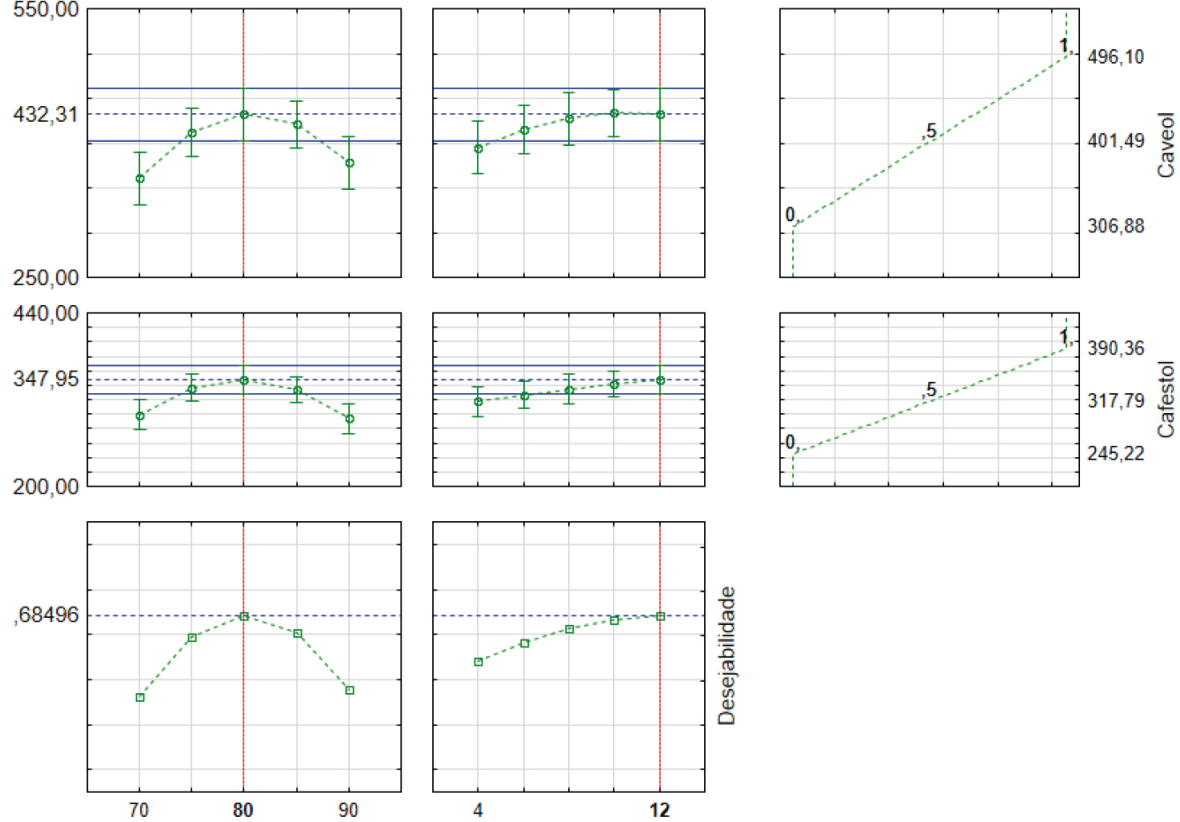

Figura 4. Desejabilidade para caveol e cafestol obtidos por saponificação em micro-ondas e determinados por CLAE

mesma temperatura, variando-se o tempo de 5 a 100 min (Figura 5B). Observou-se comportamento similar para os dois compostos, tanto no tratamento por micro-ondas quanto em banho térmico.

O uso de tempos superiores a 12 min não foi eficiente para a saponificação em micro-ondas, havendo decréscimo no teor dos diterpenos em tempos mais prolongados. Em processos extrativos, durante o aquecimento por micro-ondas, devido à temperatura e à pressão altamente localizadas, ocorre migração seletiva de compostos a uma taxa mais rápida, ${ }^{27,41}$ porém, embora as ondas eletromagnéticas sejam distribuídas uniformemente em função do aumento da temperatura reacional, em sistemas heterogêneos pode ocorrer o fenômeno de hot spots. ${ }^{42,43}$ Este fenômeno consiste na formação de zonas irregulares de aquecimento, que podem estar relacionadas com a degradação dos compostos. Chen et al. ${ }^{44}$ observaram que, mesmo em temperaturas fixas, tempos mais prolongados de irradiação das micro-ondas provocaram a degradação de saponinas.

Comparando-se os perfis das reações de saponificação em micro-ondas (Figura 5A) e em banho térmico (Figura 5B), verificou-se que em ambos os casos há um aumento do conteúdo de caveol e cafestol até o tempo ótimo de reação (12 min para micro-ondas, 60 min para banho), seguido da redução dos diterpenos. A redução do tempo de reação verificado para a saponificação em micro-ondas deve-se a uma maior taxa de extração em comparação a reação em banho termostático (Material Suplementar, Figura 2S).

$\mathrm{O}$ tempo teve maior influência para reação conduzida em banho térmico do que para a reação em micro-ondas (Figura 5B). Aproximadamente $80 \%$ a mais dos diterpenos foram obtidos em 60 minutos do que em 40 minutos de reação. O tempo ótimo (60 min) de processo corrobora com o preconizado por Dias et al. ${ }^{18}$

Com relação à eficiência do processo, menores teores de caveol e cafestol foram obtidos pela reação de saponificação em micro-ondas $(24 \%$ e $35 \%$, respectivamente) comparativamente ao método de saponificação em banho. O menor rendimento, notadamente do cafestol, pode estar associado a uma menor eficiência no processo de saponificação ou a degradação térmica do composto.

Destaca-se, no entanto, que houve redução significativa do tempo (1/5 em relação ao aquecimento convencional), e que o processo em micro-ondas permitiu uma ampliação expressiva de escala (5 vezes). Segundo Zhou et al.,${ }^{30}$ reações químicas conduzidas em micro-ondas ocorrem mais rapidamente devido à redução da energia de ativação da reação. Souza et al. ${ }^{45}$ também descrevem que na saponificação por micro-ondas para obtenção de colesterol e óxidos de colesterol em camarão houve redução do tempo (75\%) em relação ao método de saponificação a frio.
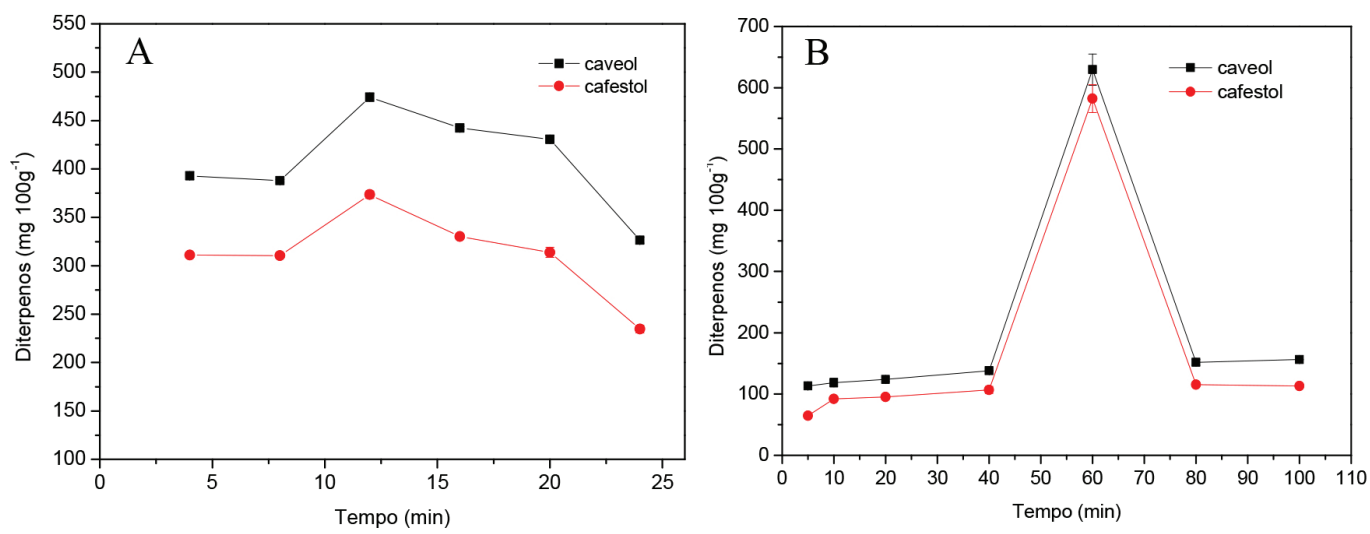

Figura 5. Perfil da concentração de caveol e cafestol obtidos por saponificação em micro-ondas (A) e em banho térmico (B) a $80^{\circ} \mathrm{C}$ em função do tempo de reação 


\section{CONCLUSÃO}

A temperatura e o tempo influenciaram significativamente a reação de saponificação por micro-ondas para extração de caveol e cafestol em café arábica torrado. A melhor condição ocorreu a $80^{\circ} \mathrm{C}$ durante $12 \mathrm{~min}$.

Utilizando a saponificação em micro-ondas obteve-se menor rendimento dos diterpenos, mas houve redução expressiva do tempo de processo em comparação a reação conduzida em banho térmico, sendo possível a ampliação da escala para extração com maior massa de amostra. A técnica de reação de saponificação em micro-ondas foi aplicada com sucesso, podendo ser considerada uma alternativa interessante para extração acelerada de diterpenos de café torrado.

\section{MATERIAL SUPLEMENTAR}

No material suplementar de livre acesso, disponível em http:// quimicanova.sbq. org.br, constam os espectros de UV-vis do caveol e cafestol (Figura 1S), o gráfico de taxa de extração para saponificação em micro-ondas e em banho térmico (Figura 2S), e as Tabelas $1 \mathrm{~S}$ e $2 \mathrm{~S}$ exibem o resultado da Análise de Variância (Anova) para o modelo polinomial de segunda ordem que descreve a significância da temperatura e do tempo na reação de saponificação por micro-ondas para extração de caveol e cafestol em café arábica torrado.

\section{AGRADECIMENTOS}

Os autores agradecem ao Conselho Nacional de Desenvolvimento Científico e Tecnológico $(\mathrm{CNPq})$ pelo suporte financeiro, ao Departamento de Química da Universidade Estadual de Londrina, em especial a equipe do Laboratório DIA (Laboratório de Desenvolvimento de Instrumentação e Automação Analítica), a Profa. Dra. Suzana Lucy Nixdorf, a Dra. Alexandra Maffei Monteiro e ao Prof. Dr. César Tarley pela disponibilização dos equipamentos.

\section{REFERENCIAS}

1. http://www.ico.org/prices/new-consumption-table.pdf. Acessado em agosto 2017.

2. http://www.conab.gov.br/OlalaCMS/uploads/arquivos/17_01_17_14_51_54_ boletim_cafe_-_janeiro_de_2017.pdf. Acessado em agosto 2017.

3. Higdon, J. V; Frei, B.; Crit. Rev. Food Sci. Nutr. 2006, 46, 10; Butt, M. S.; Sultan, M. T. Crit Rev Sci Nutr. 2011, 51, 363; Toci, A. T.; Neto, V. J. M. F.; Torres, A. G.; Farah, A.; LWT -- Food Sci. Technol. 2013, 50, 581.

4. Freedman, N. D.; Park, Y.; Abnet, C. C.; Hollenbeck, A. R.; Sinha, R. Nejm. 2012, 1; Ding, M.; Satija, A.; Bhupathiraju, S. N.; Hu, Y.; Sun, Q.; Han, J.; Lopez-Garcia, E.; Willett, W.; Van Dam, R. M.; Hu, F. B.; Circulation. 2015, 132, 2305.

5. Pettitt, B. C.; J. Agric. Food Chem. 1987, 35, 549.

6. Speer, K.; Kolling-Speer, I. T.; Braz. J. Plant Physiol. 2006, 18, 201.

7. George, S. E.; Ramalakshmi, K.; Rao, L. J. M.; Crit. Rev. Food Sci. Nutr. 2008, 48, 464 .

8. Cavin, C.; Holzhaeuser, D.; Scharf, G.; Constable, A.; Huber, W. W.; Schilter, B.; Food Chem. Toxicol. 2002, 40, 1155; Ludwig, I. A.; Clifford, M. N.; Lean, M. E. J.; Ashihara, H.; Crozier, A.; Food Funct. 2014, 5, 1695; Gaascht, F.; Dicato, M.; Diederich, M. Genes Nutr. 2015, 10, 1; Campanha, F. G.; Dias R. C. E.; Benassi, M. T.; Coffee Sci. 2010, 5,87 .

9. Guercia, E.; Berti, F.; Navarini, L.; Demitri, N.; Forzato, C.; Tetrahedron: Asymmetry. 2016, 27, 649.

10. Oigman, S. S.; De Souza, R. O. M. A.; Dos Santos Júnior, H. M.; Hovell, A. M. C.; Hamerski, L.; Rezende, C. M.; Food Chem. 2012, 134, 999.
11. Benassi, M. T.; Dias, R. C. E. Em Coffee in health and disease prevention; Preedy, V. R, eds.; Elsevier, 2015, cap. 109.

12. Kolling-Speer, I.; Strohschneider, S.; Speer, K.; J. High Resolut. Chromatogr. 1999, 22, 43.

13. Scharnhop, H.; Winterhalter, P.; J. Food Compos. Anal. 2009, 22, 233.

14. Zhang, C.; Linforth, R.; Fisk, I. D.; Food Res. Int. 2012, 49, 27.

15. Tsukui, A.; Santos Júnior, H. M.; Oigman, S. S.; De Souza, R. O. M. A.; Bizzo, H. R.; Rezende, C. M.; Food Chem. 2014, 164, 266.

16. Urgert, R.; Van Der Weg, G.; Kosmeijer-Schuil, T. G.; Van De Bovenkamp, P.; Hovenier, R.; Katan, M. B.; J. Agric. Food Chem. 1995, 43, 2167

17. Roos, B.; Van der Weg, G.; Urgert, R.; Van de Bovenkamp, P.; Charrier, A.; Katan, M. B.; J. Agric. Food Chem. 1997, 45, 3065.

18. Dias, R. C. E.; Campanha, F. G.; Vieira, L. G. E.; Ferreira, L. P.; Pot, D.; Marraccini, P.; Benassi, M. T.; J. Agric. Food Chem. 2010, 58, 88.

19. Acevedo, F.; Rubilar, M.; Scheuermann, E.; Cancino, B.; Uquiche, E.; Garcés, M.; Inostroza, K.; Shene, C.; J. Biobased Mater. Bioenergy 2013, 7, 420

20. Dias, R. C. E.; Faria, A. F.; Mercadante, A. Z.; Bragagnolo, N.; Benassi, M. T.; J. Braz. Chem. Soc. 2013, 24, 492.

21. Barbosa, H. M. A.; Melo, M. M. R.; Coimbra, M. A.; Passos, C. P.; Silva, C. M.; J. Supercrit. Fluids. 2014, 85, 165.

22. Mori, A. L. B.; Kalschene, D. L.; Ferrão, M .A. G.; Fonseca, A. F. A; Ferrão, R. G.; Benassi, M. T.; J. Food Compos. Anal. 2016, 52, 52.

23. Bhattacharya, M.; Basak, T.; Energy. 2016, 97, 306.

24. Bengis, R.; Anderson, R. J. T.; J. Biol. Chem. 1932, 97, 99.

25. Corey, E. J.; Wess, G.; Xiang, Y. B.; Singh, A. K.; J. Am. Chem. Soc. 1987, 109, 4717.

26. Xiao, X.; Song, W.; Wang, J.; Li, G.; Anal. Chim. Acta. 2012, 712, 85.

27. Upadhyay, R.; Ramalakshmi, K.; Jagan Mohan Rao, L.; Food Chem. 2012, 130, 184 .

28. Wang, H.; Ding, J.; Ren, N.; TrAC - Trends Anal. Chem. 2016, 75, 197.

29. Sá, M. M.; Meier, L.; Dutra, L. G.; Bisol, T. B.; Rev. Virtual Química. 2010, 2,165

30. Zhou, J.; Xu, W.; You, Z.; Wang, Z.; Luo, Y.; Gao, L.; Yin, C.; Peng, R.; Lan, L.; Sci. Rep. 2016, 6, 25149.

31. Piñeiro-Avila, G.; Salvador, A.; De La Guardia, M.; Anal. Chim. Acta. 1998, 371, 297.

32. Uquiche, E.; Jeréz, M.; Ortiz, J.; Innov. Food Sci. Emerg. Technol. 2008, 9, 495.

33. Statistica for Windows software. V. 7.1, Tulsa, OK, USA, 2007.

34. Campanha, F. G.; Dias, R. C. E.; Benassi, M. T.; Coffee Sci. $2010,87$.

35. Kitzberger, C. S. G.; Scholz, M. B. dos S.; Pereira, L. F. P.; Benassi, M. T.; Pesqui. Agropecu. Bras. 2013, 48, 1498

36. Kitzberger, C. S. G.; Scholz, M. B. dos S.; Benassi, M. T.; Food Res. Int. 2014, 61, 61 .

37. Li, J.; Zu, Y. G.; Fu, Y. J.; Yang, Y. C.; Li, S. M.; Li, Z. N.; Wink, M.; Innov. Food Sci. Emerg. Technol. 2010, 11, 637.

38. Dias, R. C. E.; Faria-Machado, A. F.; Mercadante, A. Z.; Bragagnolo, N.; Benassi, M. T.; Eur. Food Res. Technol. 2014, 239, 961.

39. Chartier, A.; Beaumesnil, M.; Oliveira, A. L.; Elfakir, C.; Bostyn, S.; Talanta 2013, 117, 102.

40. Liu, H.; Shao, J.; Li, Q.; Li, Y.; Yan, H. M.; He, L.; J. AOAC Int. 2012, 95, 1138.

41. Spigno, G.; De Faveri, D. M.; J. Food Eng. 2009, 93, 210.

42. Hoz, A. de la; Diaz-Ortiz, A.; Moreno, A.; Chem. Soc. Rev. 2005, 34, 164.

43. Horikoshi, S.; Osawa, A.; Abe, M.; Serpone, N.; J. Phys. Chem. C 2011, 115,23030 .

44. Chen, Y.; Xie, M. Y.; Gong, X. F.; J. Food Eng. 2007, 81, 162.

45. Souza, H. A. L.; Mariutti, L. R. B.; Bragagnolo, N.; J. Steroid Biochem. Mol. Biol. 2017, 169, 88. 